\title{
Das Reden der Neulinge und andere Sünden. Parlamentarische Sozialisation und Parlamentskultur in zwei deutschen Landtagen
}

\author{
Marion Reiser, Claudia Hülsken, Bertram Schwarz und Jens Borchert
}

\begin{abstract}
"The nostalgic talk of the older senators about the unhappy lot of the freshman in the good old days is one way the senior senators keep the younger men in place. One freshman Democrat, for example, after a floor speech found himself sitting next to Senator George, than Dean of the Senate. Thinking that he should make polite conservation, the freshman asked the Georgia patriarch what major changes had taken place in the Senate during his long service. Senator George replied, ,Freshmen didn't use to talk so much."'1

„Das war die erste Fraktionssitzung und einer der Neuen ist aufgestanden und hat sich zu Wort gemeldet. Danach sagte ein älterer Kollege: „Hat dem eigentlich keiner gesagt, dass man sich als Neuer hier nicht zu Wort zu melden hat, es sei denn man stellt die Frage: ,Will jemand 'ne Tasse Kaffee?"“2
\end{abstract}

Was - außer der bloßen Tatsache seiner Wahl - macht den Abgeordneten ${ }^{3}$ zum Abgeordneten? Wie wird aus Menschen beiderlei Geschlechts, unterschiedlicher regionaler Herkunft, mit verschiedenen Bildungs- und Berufserfahrungen und nicht zuletzt mit teilweise ganz gegensätzlichen politischen Auffassungen eine Gruppe von Parlamentariern, die in wesentlichen Punkten gleicher Auffassung sind? Und wie werden diese geteilten Werte und Normen vermittelt? Typischerweise herrscht in Parlamenten weitgehende Übereinstimmung darüber, was die Rolle der Parlamentarier, was die Aufgaben und Entscheidungsmechanismen des Parlamentes, was die „Würde des Hohen Hauses“ und was angebrachte und unangebrachte Verhaltensweisen angeht.

Dieser parteiübergreifende Konsens manifestiert sich immer dann besonders klar, wenn er durch das Verhalten einzelner Abgeordneter oder ganzer Fraktionen in Frage gestellt wird. Das betrifft triviale Dinge wie Bekleidungsfragen ebenso wie grundlegende Regeln der politischen Auseinandersetzung. Unter den nordrhein-westfälischen Landtagsabgeordneten (fast) aller Fraktionen gab es helle Empörung, als die SPD im Juni 2011 bei der Abstimmung zur West-LB zum zweiten Mal innerhalb kurzer Zeit die Pairing-Übereinkunft mit der CDU missachtete, eine eigentlich krank gemeldete Abgeordnete überraschend im Landtag erscheinen ließ und der Landesregierung so - zumindest theoretisch - eine knappe Mehrheit sicherte. ${ }^{4}$ Diese bewusste Verletzung einer der grundlegenden und in den meisten

1 Donald R. Matthews, The Folkways of the United States Senate, in: American Political Science Review, 53. Jg. (1959), H. 4, S. 1064 - 1089, S. 1066.

2 Interview mit einem Abgeordneten des Landtags von Baden-Württemberg (BaWü-NI-38). Die im Rahmen dieser Studie geführten Leitfadeninterviews wurden anonymisiert. Interviews mit Abgeordneten des Landtags Baden-Württemberg beginnen im Folgenden mit dem Kürzel BaWü, solche mit Abgeordneten des Landtags Nordrhein-Westfalen mit dem Kürzel NRW.

3 Zugunsten der Lesbarkeit wurde auf die weibliche Form verzichtet. Wir meinen stets weibliche und männliche Abgeordnete.

4 In den Medien finden sich hier leicht abweichende Darstellungen. Vgl. Barbara Schmidt-Mattern, Schüsse, die nach hinten losgehen. Die NRW-SPD und das Abstimmungs-Desaster, in: Deutschland Funk vom 7. Juli 2011, http://www.dradio.de/dlf/sendungen/dlfmagazin/1500014/ (Abruf 
Parlamenten gepflegten Fairness-Regeln - keine Fraktion soll durch die Erkrankung eines Abgeordneten einen Stimmenvorteil haben - erregte Missfallen nicht nur bei der Opposition, die extra einen ihrer Abgeordneten beurlaubt hatte, sondern auch beim grünen Regierungspartner und erzeugte auch bei altgedienten wie neuen Sozialdemokraten erhebliches Unbehagen. ${ }^{5}$ Im Ergebnis musste die parlamentarische Geschäftsführerin der SPD-Fraktion einen Fehler einräumen und ihr Amt abgeben - wodurch die Gültigkeit der Norm eindrucksvoll bestätigt wurde.

Organisationen prägen ihre Mitglieder. Wie aber vollzieht sich dieser Prozess in einer Institution, deren Angehörige von verschiedenen Organisationen rekrutiert und dann von den Wählern entsandt wurden? Politikwissenschaftler haben sich in der Vergangenheit immer wieder mit dieser Frage beschäftigt. Die klassische Untersuchung von Donald Matthews zum US-amerikanischen Senat der 1950er Jahre förderte ein recht striktes Normensystem zutage, das Neulingen in einer Lehrzeit, die sie überwiegend schweigend verbringen sollten, vermittelt wurde. ${ }^{6}$ Ganz so hart wie in dem von Matthews wiedergegebenen Beispiel - bei dem in der Anekdote zurechtgewiesenen Neuling handelte es sich übrigens um John F. Kennedy - sind die Anpassungsprozesse heute sicherlich nicht mehr. Aber die gleichfalls oben zitierte Aussage eines neuen Abgeordneten in Baden-Württemberg verweist doch darauf, dass die Norm anfänglicher Zurückhaltung für Neulinge auch heute noch eine gewisse Gültigkeit hat.

Wenn man die Frühzeit der Grünen in den deutschen Parlamenten mit dem gegenwärtigen Auftreten der Abgeordneten dieser Partei vergleicht, kann man jedenfalls von einem wirkungsmächtigen parlamentarischen Sozialisationsprozess ausgehen. Nach den ersten Wahlerfolgen der Grünen wurde zum Beispiel das Brechen der Kleiderkonventionen in den Landtagen Anfang der 1980er Jahre noch als bewusste Provokation der bestehenden Parlamentskultur inszeniert, von den anderen Fraktionen auch so wahrgenommen und dementsprechend sanktioniert. In der Folge mussten die Grünen die Gültigkeit des Verdiktes „violators will be less effective legislators"7 anerkennen. Die Einsicht, dass ein bewusstes Abweichen von den Normen die Möglichkeiten für die grünen Abgeordneten verringerte, ihre politischen Inhalte umzusetzen, führte zu Lernprozessen und damit zu einer sichtbaren Anpassung in der Form. ${ }^{8}$ Im Verlauf des parlamentarischen Sozialisationsprozesses werden die Neulinge also individuell wie kollektiv mit den institutionell vorherrschenden Normen und Verhaltensweisen vertraut gemacht und verinnerlichen diese.

am 24. November 2011); Reiner Burger, Inland in Kürze - Britta Altenkamp tritt zurück, in: FAZ vom 4. Juli 2011, S. 4; Bernd Dörries, Nachspiel in WestLB-Affäre, in: SZ vom 6. Juli 2011, S. 6.

5 Vgl. unter anderem NRW-NII-44; NRW-NII-4; NRW-NII-10; NRW-NII-20; NRW-NII-25; NRW-NII-39.

6 Vgl. Donald R. Matthews, a.a.O. (Fn. 1); ders., U.S. Senators and Their World, Chapel Hill 1960.

7 Charles G. Bell / Charles M. Price, The First Term: A Study of Legislative Socialization, Beverly Hills / London 1975, S. 71.

8 Vgl. Lilian Klotzsch / Klaus Könnemann / Jörg Wischermann / Bodo Zeuner, Zwischen Systemopposition und staatstragender Funktion: Die Grünen unter dem Anpassungsdruck parlamentarischer Mechanismen, in: Dietrich Herzog / Bernhard Weßels (Hrsg.), Konfliktpotentiale und Konsensstrategien, Opladen 1989, S. 180 - 215; Renate Mayntz / Friedhelm Neidhardt, Parlamentskultur: Handlungsorientierungen von Bundestagsabgeordneten - eine empirisch explorative Studie, in: ZParl, 20. Jg. (1989), H. 3, S. 370 - 387; Thomas Poguntke, Alternative Politics. The German Green Party, Edinburgh 1993. 
Darüber, wie dieser Sozialisationsprozess heute in deutschen Parlamenten abläuft, wissen wir allerdings vergleichsweise wenig. Ziel des Projektes „Parlamentarische Sozialisation von Landtagsabgeordneten“, dessen erste Ergebnisse dieser Beitrag vorstellt, ist es, diesen Zustand zu ändern. ${ }^{9}$ Daher werden die 2010 beziehungsweise 2011 neu gewählten Abgeordneten der Landtage von Nordrhein-Westfalen und Baden-Württemberg in einer qualitativen Paneluntersuchung insgesamt dreimal befragt - vor ihrer Wahl, ein Jahr nach ihrer Wahl und nach Ende ihrer ersten Wahlperiode. Die ersten beiden Befragungswellen in Nordrhein-Westfalen und die erste Welle in Baden-Württemberg sind bereits abgeschlossen. Als Kontrollgruppe und als Vermittler der vorherrschenden Parlamentskultur wurde zudem jeweils eine Gruppe von erfahrenen Landtagsabgeordneten befragt. Hier werden erste Erkenntnisse zu den Vorerfahrungen und Erwartungen der neuen Abgeordneten und (für Nordrhein-Westfalen) zu den ersten Sozialisationseffekten sowie zur jeweils gültigen Parlamentskultur präsentiert. Diese fokussieren auf die Ziele und Erwartungen der neuen Abgeordneten, die durch die Parlamentskultur definierten Spielregeln und Normen sowie auf die Sozialisationsagenten als Vermittler der Parlamentskultur.

\section{Parlamentarische Sozialisation von Neuparlamentariern}

Nach jeder Parlamentswahl zieht in Deutschland ungefähr jeder dritte Abgeordnete erstmals in die Parlamente auf Landes-, Bundes- oder Europaebene ein. Obwohl die überwiegende Mehrheit vor dem Einzug in das Parlament bereits politische Erfahrungen in lokalen und regionalen Parteifunktionen und öffentlichen Ämtern im Rahmen der „Ochsentour“10 gesammelt hat, haben die Neuen dennoch nur eine vage Vorstellung davon, was ein Abgeordneter zu tun und wie er sich zu verhalten hat. ${ }^{11}$ Damit sind nicht nur die formellen Regeln und Tätigkeiten im hocharbeitsteiligen Parlament gemeint, sondern insbesondere die informellen Verhaltenserwartungen und Normvorstellungen, die das eigene und das kollegiale Verhalten gegenüber den anderen Fraktions- und Parlamentsmitgliedern steuern. ${ }^{12}$ So gibt es im Rahmen der Parlamentarismusforschung schon lange die Erkenntnis, dass sich in Parlamenten ebenso wie in anderen Institutionen im Laufe der Zeit eine eigene Kultur herausbildet, die über Wahlperioden hinweg existiert. ${ }^{13}$ Die neuen Abgeordneten müssen sich mit diesen informellen Verhaltenserwartungen und Werthaltungen auseinandersetzen und sich auf sie einstellen, um in das Parlament integriert zu werden und als vollwertiges und kompetentes Mitglied zu gelten. ${ }^{14}$ Die grundsätzliche Annahme lautet, dass die neuen Abgeordneten die parlamentarische Kultur mit ihren Arbeitsweisen, Routi-

9 Das Projekt wird durch die Fritz Thyssen Stiftung gefördert.

10 Dietrich Herzog, Politische Karrieren: Selektion und Professionalisierung politischer Führungsgruppen, Opladen 1975, S. 159, S. 221.

11 Vgl. Werner J. Patzelt, Abgeordnete und ihr Beruf. Interviews, Umfragen, Analysen, Berlin 1995; Ulrich Sarcinelli, Parlamentarische Sozialisation in der Bundesrepublik, in: ZParl, 20. Jg. (1989), H. 3, S. $388-407$.

12 Vgl. Renate Mayntz / Friedhelm Neidhardt, a.a.O. (Fn. 8).

13 Vgl. Donald R. Matthews, a.a.O. (Fn. 1); Renate Mayntz / Friedhelm Neidhardt, a.a.O. (Fn. 8); Helmar Schöne, Alltag im Parlament, Parlamentskultur in Theorie und Empirie, Baden-Baden 2010.

14 Vgl. Werner J. Patzelt, Parlamentssoziologie, in: Viktoria Kaina / Andrea Römmele (Hrsg.), Politische Soziologie, Wiesbaden 2009, S. 311 - 351. 
nen und Spielregeln allmählich und eher unbewusst erwerben. Dieser Lern- und Anpassungsprozess wird als parlamentarische Sozialisation bezeichnet. ${ }^{15}$

Die Analyse dieser Sozialisationsprozesse ist dabei aus drei Perspektiven relevant: So wird die Anpassung an die zentralen Normen und damit die Integration der Elite erstens im Rahmen der Eliten- und Parlamentarismusforschung als wichtige Voraussetzung für die Funktionsfähigkeit des Parlaments diskutiert. Der Grundkonsens über diese Regeln und Normen ermögliche trotz der gesellschaftlichen und parteipolitischen Differenzen kooperative Konfliktaustragung und Interessenausgleich. ${ }^{16}$

Zweitens ist die Analyse auch unter Repräsentationsaspekten höchst bedeutsam. In einer weiten Definition fällt unter die Sozialisation der Abgeordneten nicht nur der Sozialisationskontext innerhalb, sondern auch außerhalb des Parlaments. ${ }^{17}$ So gehört zur Rolle und zu den charakteristischen Aufgaben der Parlamentarier auch die Arbeit im Wahlkreis, an der Parteibasis und bei Interessenverbänden, wo die Abgeordneten auch mit unterschiedlichen Rollenerwartungen konfrontiert werden. Der einzelne Abgeordnete steht - auch aufgrund der durch das enge Zeitbudget notwendigen Prioritätensetzung - im Spannungsfeld zwischen seinen Aufgaben und den Erwartungen im Parlament selbst und jenen, die von außerhalb an ihn herangetragen werden. Die parlamentarische Sozialisation ist drittens individuell für den einzelnen Abgeordneten aus Karriere- und Einflussgesichtspunkten relevant. ${ }^{18}$ So ist „eine gewisse Anpassungsbereitschaft an (...) die innerparlamentarischen und innerparteilichen Apparate (...) wohl der unabdingbare Preis für eine erfolgreiche Tätigkeit im Parlament "19 und für die Wiedernominierung in der lokalen Parteiorganisation. ${ }^{20}$

Obwohl in vielfältigen Abhandlungen daher auf die große Bedeutung der parlamentarischen Sozialisationsprozesse hingewiesen wurde ${ }^{21}$, gibt es für Deutschland, aber auch in der international vergleichenden Forschung nur einen vergleichsweise geringen Forschungsstand. So existieren für deutsche Parlamente nur sehr wenige empirische Untersuchungen, die die Parlamentskultur ${ }^{22}$ in den Blick nehmen. Sozialisationsprozesse der Neuparlamentarier wurden explizit nur in einer Panelstudie von Bernhard Badura und Jürgen Reese ${ }^{23}$ auf Basis von 49 Jungparlamentariern im Deutschen Bundestag analysiert. Als zentrale Ergeb-

15 Vgl. Helmar Schöne, Probleme und Chancen parlamentarischer Integration. Eine empirische Studie zum Ost-West-Integrationsprozess unter Abgeordneten, Wiesbaden 1999, S. 5; Ulrich Sarcinelli, a.a.O. (Fn. 11), S. 391; Bernhard Badura / Jürgen Reese, Jungparlamentarier in Bonn ihre Sozialisation im Deutschen Bundestag, Stuttgart 1976, S. 32 - 38.

16 Vgl. Dietrich Herzog / Hilke Rebenstorf / Bernhard Weßels (Hrsg.), Parlament und Gesellschaft - Eine Funktionsanalyse der repräsentativen Demokratie, Opladen 1993; Helmar Schöne, a.a.O. (Fn. 15).

17 Vgl. Ulrich Sarcinelli, a.a.O. (Fn. 11).

18 Vgl. Bernhard Badura / Jürgen Reese, a.a.O. (Fn. 15), S. 108.

19 Ulrich Sarcinelli, a.a.O. (Fn. 11), S. 389. Vgl. auch Werner J. Patzelt, Die Integration ostdeutscher Abgeordneter in "gesamtdeutsche" Parlamente, in: ZParl, 31. Jg. (2000), H. 3, S. $542-568$.

20 Vgl. Bodo Zeuner, Kandidatenaufstellung zur Bundestagswahl 1965: Untersuchung zur innerparteilichen Willensbildung und zur politischen Führungsauslese, Den Haag 1970.

21 Vgl. Donald R. Matthews, a.a.O. (Fn. 6); Anthony Mughan / Janet Box-Steffensmeier / Roger Scully, Mapping Legislative Socialisation, in: European Journal of Political Research, 32. Jg. (1997), H. 1, S. 93 - 106; Werner J. Patzelt, a.a.O. (Fn. 19); Ulrich Sarcinelli, a.a.O. (Fn. 11); Dietrich Herzog / Hilke Rebenstorf / Bernhard Weßels (Hrsg.), a.a.O. (Fn. 16).

22 Vgl. Renate Mayntz / Friedhelm Neidhardt, a.a.O. (Fn. 8); Helmar Schöne, a.a.O. (Fn. 13); Manfred Schwarzmeier, Parlamentarische Mitsteuerung. Strukturen und Prozesse informalen Einflusses im Deutschen Bundestag, Wiesbaden 2001.

23 Vgl. Bernhard Badura / Jürgen Reese, a.a.O. (Fn. 15). 
nisse stellten sie eine Konvergenz bestimmter parlamentsbezogener Einstellungen bei den Neulingen sowie eine zunehmende Konzentration auf parlamentsinterne Vorgänge fest. ${ }^{24}$

Für die vorliegende Analyse der frühen parlamentarischen Sozialisationsphase sind insbesondere drei Aspekte wichtig, die im Rahmen dieses Beitrags näher beleuchtet werden:

Erstens ist es von zentralem Interesse, welche parlamentsbezogenen Einstellungen und Handlungsorientierungen die neuen Abgeordneten als Ergebnis der vorparlamentarischen Erfahrungen mitbringen und welche Ziele sie sich für ihre inhaltliche Arbeit setzen. Neuparlamentarier in deutschen Parlamenten sind überwiegend keine politischen Neulinge. ${ }^{25}$ Sie haben überwiegend eine mehrjährige Sozialisation in ihrer Partei und in kommunalen Parlamenten erfahren. Dietrich Herzogs Arbeiten weisen darauf hin, dass es im Zuge der Karrierisierung und Professionalisierung unter den Parlamentariern zu einer fortdauernden Anpassung an die Normen und Werte des politischen Mandats kommt. ${ }^{26}$ Entsprechend kann vermutet werden, dass die Neuparlamentarier aufgrund der jeweils spezifischen vorparlamentarischen Sozialisationsprozesse mit unterschiedlichen Erwartungen und Vorstellungen zur politischen und parlamentarischen Arbeit in das Parlament einziehen. Welche dies aber sind und welche Ziele sie sich im Vorfeld setzen, ist nicht bekannt.

Ein zweiter wichtiger Aspekt sind die informellen Spielregeln, Normen und Verhaltensweisen in den deutschen Parlamenten, die sich die neuen Abgeordneten aneignen. Empirische Erkenntnisse, welche Normen und Verhaltenserwartungen für die deutschen (Landes-) Parlamente prägend sind, gibt es bisher jedoch nach wie vor kaum. Zu nennen ist hier insbesondere die explorative Studie von Renate Mayntz und Friedhelm Neidhardt zum Deutschen Bundestag sowie die aktuelle Untersuchung von Helmar Schöne zum Bundestag und zum Sächsischen Landtag. ${ }^{27}$ Sie finden in diesen Parlamenten dabei sehr ähnliche Spielregeln und Verhaltensstandards, wie sie Matthews für den US-Senat herausgearbeitet hat. ${ }^{28}$ So stellten Mayntz und Neidhardt ein gemeinsames Verständnis der Abgeordneten von der „Würde des Hohen Hauses“ fest, die sie ebenso wie die Unabhängigkeit des Parlaments verteidigen. ${ }^{29}$ Darunter werden zentrale Normen und Erwartungen gefasst, wie man sich im Parlament verhalten soll, wie beispielsweise Kleiderfragen, der Debatten- und der Sprachstil.

24 Im Zuge der Wiedervereinigung entstanden zwar einige Studien, die sich mit der Integration ostdeutscher Abgeordnete in die gesamtdeutschen Parlamente beschäftigen, vgl. Helmar Schöne, a.a.O. (Fn. 15); ders., Vereinheitlichung und Diversität: Elitenintegration im Abgeordnetenhaus von Berlin, in: ZParl, 31. Jg. (2000), H. 3, S. 569 - 584; Werner J. Patzelt, a.a.O. (Fn. 19); Susanne Könen, Wo sind die Rebellen hin? Dissentierendes Abstimmungsverhalten in ost- und westdeutschen Landtagen, Wiesbaden 2009. Die Erkenntnisse sind aufgrund der besonderen Bedingungen und der unterschiedlichen politischen Sozialisation der Abgeordneten jedoch nur eingeschränkt aussagekräftig für die „normalen“ parlamentarischen Sozialisationsprozesse von Neuparlamentariern.

25 Vgl. Dietrich Herzog / Hilke Rebenstorf / Camilla Werner / Bernhard Weßels, Abgeordnete und Bürger, Opladen 1990; Jens Borchert / Klaus Stolz, Die Bekämpfung der Unsicherheit: Politikerkarrieren und Karrierepolitik in der Bundesrepublik Deutschland, in: PVS, 44. Jg. (2003), H. 2, S. 148 - 173; Michael Edinger / Lars Vogel, Role Perceptions, Party Cohesion and Political Attitudes of East and West German Parliamentarians, in: Czech Sociological Review, 41. Jg. (2005), H. 3, S. 375 - 399; Heinrich Best / Stefan Jahr, Politik als prekäres Beschäftigungsverhältnis: Mythos und Realität der Sozialfigur des Berufspolitikers im wiedervereinten Deutschland, in: ZParl, 37. Jg. (2006), H. 1, S. $63-79$.

26 Vgl. Dietrich Herzog, a.a.O. (Fn. 10).

27 Vgl. Renate Mayntz / Friedhelm Neidhardt, a.a.O. (Fn. 8); Helmar Schöne, a.a.O. (Fn. 13).

28 Vgl. Donald R. Matthews, a.a.O. (Fn. 1).

29 Vgl. Renate Mayntz / Friedhelm Neidhardt, a.a.O. (Fn. 8). 
Bedeutsam sind zudem Normen, die den Umgang zwischen den Parlamentariern regeln, wie Respekt, Verlässlichkeit, Kooperationsbereitschaft und Loyalität. ${ }^{30}$ Abweichungen und Verstöße gegen diese zentralen Verhaltensnormen werden sanktioniert, wie die oben angeführten Beispiele der Grünen und die Nichteinhaltung des Pairing-Abkommens zeigen. Ebenso wie im US-Senat besteht nach der Studie von Mayntz und Neidhardt auch im Bundestag eine „Unauffälligkeitserwartung“ gegenüber Parlamentsneulingen. Diese sollen sich in ihrer Lehrzeit im Hintergrund halten und nicht mit dienstälteren Abgeordneten um Aufmerksamkeit, Posten und Sitzplätze konkurrieren. ${ }^{31}$ Im Vergleich zum US-Senat haben in den deutschen Parlamenten fraktionsbezogene informelle Normen jedoch einen deutlich höheren Stellenwert. Diese umfassen dabei nicht nur die Fraktionsdisziplin, sondern auch das Nicht-Überschreiten des eigenen Kompetenzbereichs, gegenseitige Unterstützung und kollegiales Verhalten. ${ }^{32}$

Von zentralem Interesse ist drittens, wie diese Normen und Verhaltensregeln an die neuen Abgeordneten vermittelt werden: „There must always be some way for new members to learn how they are expected to behave, what they can expect of others, the limits of these rules and roles, and the penalties for violation."33 Wie Studien zeigen, werden die informellen Spielregeln und Verhaltenserwartungen insbesondere durch die Kommunikation und Interaktion zwischen den Parlamentariern vermittelt. ${ }^{34}$ Charles G. Bell und Charles M. Price unterscheiden zum Beispiel zwischen formellen und informellen Sozialisationsagenten. ${ }^{35}$ Erstere sind solche, die speziell zur Unterstützung des Sozialisierungsprozesses eingerichtet werden, beispielsweise Orientierungsprogramme für Neuparlamentarier. Diese sind allerdings zumeist auf formale Regeln gerichtet, wie sie sich etwa in der Geschäftsordnung finden, und sind für die Analyse der Parlamentskultur und der Sozialisation weniger entscheidend. Bedeutsamer für die Vermittlung der Parlamentskultur sind die informellen Sozialisationsagenten. Darunter verstehen Bell und Price individuelle - dazu zählen vor allem erfahrene Abgeordnete, die quasi als Paten für die Neuparlamentarier fungieren - und kollektive Sozialisationsagenten, die hinsichtlich Organisationsgrad, Formalität, Mitgliedschaft und Wichtigkeit höchst unterschiedlich sind und weitgehend mit den „Sozialisationsfeldern“ von Ulrich Sarcinelli übereinstimmen. So ist der Neuparlamentarier in „ein komplexes Geflecht innerparlamentarischer Sozialisationsfelder eingebunden: auf der horizontalen Ebene in das Plenum, in die Fraktion, in Ausschüsse, in Arbeitskreise beziehungsweise -gruppen, in landsmannschaftliche Gruppen und auch in eher informelle Zirkel und Strömungsgruppen. In vertikaler Hinsicht unterliegt er vielfältigen Abhängigkeiten einer Parlaments- und vor allem Fraktionshierarchie“36.

30 Vgl. Helmar Schöne, a.a.O. (Fn. 13), S. 198 f.

31 Vgl. Renate Mayntz / Friedhelm Neidhardt, a.a.O. (Fn. 8), S. 379; Donald R. Matthews, a.a.O. (Fn. 1).

32 Vgl. Helmar Schöne, a.a.O. (Fn. 13), S. 200; Renate Mayntz / Friedhelm Neidhardt, a.a.O. (Fn. 8), S. 379 .

33 Malcom E. Jewell / Samuel C. Patterson, The Legislative Process in the United States, New York 1966, S. 20.

34 Vgl. Werner J. Patzelt, Parlamente und ihre Symbolik. Aufriß eines Forschungsfeldes, in: ders. (Hrsg.), Parlamente und ihre Symbolik. Programm und Beispiele institutioneller Analyse, Opladen 2001, S. 39 - 78; Helmar Schöne, a.a.O. (Fn. 15).

35 Vgl. Charles G. Bell / Charles M. Price, a.a.O. (Fn. 7), S. 35 f.

36 Ulrich Sarcinelli, a.a.O. (Fn. 11), S. 399. 
Die empirischen Erkenntnisse zu den zentralen Vermittlungsinstanzen in den deutschen Parlamenten sind dabei uneinheitlich und stammen zumeist aus den 1970er und 1980er Jahren. Die Studie von Mayntz und Neidhardt zeigt eine starke Dominanz der Fraktion als Referenzgruppe anstelle des ganzen Bundestags, während die Fraktion in der Studie von Badura und Reese nur eine untergeordnete Rolle spielt. Daneben haben regionale Gruppen und Ausschüsse für die Integration der Parlamentarier auch über die Fraktionsgrenzen hinweg eine wichtige integrative Funktion. ${ }^{37}$

Insgesamt gibt es somit kaum (aktuelle) Erkenntnisse zur parlamentarischen Sozialisation. Insbesondere fehlen bisher Studien, die in der Lage sind, die individuellen Veränderungsprozesse und dadurch den Einfluss und die Wirkungen der parlamentarischen Sozialisation zu erfassen. Im Folgenden sollen erste empirischen Befunde zur parlamentarischen Sozialisation und Parlamentskultur in deutschen Landtagen präsentiert werden.

\section{Methoden}

Die empirische Untersuchung parlamentarischer Sozialisationsprozesse und -wirkungen wird in der wissenschaftlichen Diskussion als anspruchsvoll und komplex beurteilt, „because the socialisation of professional politicians is all but impossible to chart by conventional observational or survey methods" 38 . Im Zentrum der Untersuchung von Sozialisationsprozessen steht nämlich die Frage, wie sich der Sozialisationsprozess vollzieht und ob es dadurch zu individuellen Veränderungsprozessen bei den Neuparlamentariern kommt. Um diese erfassen zu können, ist eine mehrmalige Befragung derselben Abgeordneten notwendig. ${ }^{39}$ Daher werden die parlamentarischen Sozialisationsprozesse und ihr Einfluss in den Landtagen Baden-Württembergs und Nordrhein-Westfalens empirisch im Rahmen einer vergleichenden qualitativen Panelstudie untersucht. Die hier präsentierten Ergebnisse stützen sich auf zwei Befragungswellen: Die erste fand jeweils zwischen der Landtagswahl und der konstituierenden Sitzung des neugewählten Parlaments statt. Dabei wurden die neugewählten Abgeordneten zu ihren parlamentsbezogenen Einstellungen, Zielen und Erwartungen als Ergebnis ihrer vorparlamentarischen Erfahrungen sowie zu ihren Kenntnissen der formellen und informellen Spielregeln des Parlaments befragt. Die zweite Befragungswelle fand ein Jahr nach dem erstmaligen Einzug ins Parlament statt, das als das zentrale Lernund Anpassungsjahr gilt. ${ }^{40}$ Dadurch können Aussagen zum Ablauf der parlamentarischen Sozialisation sowie zum Einfluss des Sozialisationsprozesses auf die parlamentsbezogenen Einstellungen und Verhaltensweisen gemacht werden. Diese Befragungswelle ist für den Landtag Nordrhein-Westfalen bereits abgeschlossen. ${ }^{41}$

37 Vgl. Bernhard Badura / Jürgen Reese, a.a.O. (Fn. 15); Helmar Schöne, a.a.O. (Fn. 24); Werner J. Patzelt, a.a.O. (Fn. 19).

38 Anthony Mughan / Janet Box-Steffensmeier / Roger Scully, a.a.O. (Fn. 21), S. 94.

39 Vgl. ebenda; Andras M. Wüst, Zur Sozialisation von Neuparlamentariern im 15. Deutschen Bundestag, in: Oscar W. Gabriel / Bernhard Weßels / Jürgen W. Falter (Hrsg.), Wahlen und Wähler. Analysen aus Anlass der Bundestagswahl 2005, Wiesbaden 2009, S. 328 - 345, S. 342.

40 Vgl. Werner J. Patzelt, a.a.O. (Fn. 11); Charles G. Bell / Charles M. Price, a.a.O. (Fn. 7).

$41 \mathrm{Im}$ weiteren Projektverlauf ist eine dritte Befragungswelle geplant, die jeweils am Ende der ersten Wahlperiode stattfinden soll. Es wird angenommen, dass dann eine weitgehende Anpassung an die Parlamentskultur stattgefunden hat und die durch den Sozialisationsprozess modifizierten 
Die Abgeordneten wurden mittels persönlicher Leitfadeninterviews befragt, da Normen und Wertorientierungen als verinnerlichte allgemeine Standards nicht immer vollständig bewusst und damit schwer abstrakt abfragbar sind. ${ }^{42}$ Zielpersonen der Befragung waren alle Abgeordneten, die 2010 erstmals in den Landtag Nordrhein-Westfalen beziehungsweise 2011 erstmals in den Landtag Baden-Württemberg gewählt wurden. Von den insgesamt 119 neuen Abgeordneten in den beiden Landtagen wurden in der ersten Befragungswelle 95 persönlich befragt (80 Prozent). In der zweiten Welle wurden in Nordrhein-Westfalen 46 von ursprünglich 47 Teilnehmern erneut interviewt (98 Prozent).

Neben den Neuparlamentariern wurden in den beiden Landtagen bisher insgesamt 33 erfahrene Abgeordnete befragt, die dem Parlament bereits mindestens eine Wahlperiode lang angehören und entsprechend den Kriterien Zugehörigkeitsdauer zum Parlament, Parteizugehörigkeit, innerparlamentarische Funktionen sowie geographische Herkunft und Geschlecht systematisch variieren. Diese erfahrenen Abgeordneten dienen zum einen als Kontrollgruppe, zum anderen sind sie hinsichtlich ihrer Rolle als Sozialisationsagenten und Vermittler der Parlamentskultur mit ihren informellen Regeln und Verhaltenserwartungen wichtig. Insgesamt wurden bislang 186 persönliche Leitfadeninterviews geführt.

\section{Der Beginn der parlamentarischen Sozialisation: Klare Ziele - unklare Erwartungen}

Mit welchen Vorstellungen, Erwartungen und Zielen kommen neue Abgeordnete in die Landtage? Interessanterweise besteht eine deutliche Diskrepanz zwischen den Zielsetzungen einerseits und den Erwartungshaltungen andererseits, mit denen neue Abgeordnete in ihre erste Mandatszeit starten.

Nahezu jeder neue Landtagsabgeordnete zieht mit dem artikulierten Ziel in den Landtag ein, politisch gestalten zu wollen. ${ }^{43}$ Dieser recht abstrakt formulierte Gestaltungswille wird dabei zumeist durch klare inhaltliche Zielsetzungen konkretisiert. So äußern die neuen Abgeordneten spezifische Wahlkreisanliegen beziehungsweise politikfeldspezifische Ziele wie eine andere Bildungspolitik oder die Stärkung des ländlichen Raumes, die sie realisieren wollen. ${ }^{44}$ Häufig haben sie im Rahmen ihres zumeist langjährigen kommunal- beziehungsweise parteipolitischen Engagements diese inhaltlichen Ziele und Projekte nicht umsetzen können. Daher ist ein vielfach genanntes Motiv für den Wechsel von der Kommunal- in die Landespolitik insbesondere die Überwindung der kommunalpolitischen Zuständigkeitsgrenzen. ${ }^{45}$ Häufig werden neben dem Landtagsmandat die kommunalen Mandate gezielt fortgeführt, um die Verzahnung beider Ebenen zu intensivieren.

Ein weiteres Ziel und Motiv für den Wechsel in die Landespolitik ist der Reiz der konzentrierten inhaltlichen Arbeit. Daher streben die neuen Abgeordneten sehr bewusst den Wechsel vom Feierabendpolitiker zum professionellen Mandat an. Das oft so negativ konnotierte Berufspolitikerdasein wird dabei interessanterweise von etwa einem Drittel der

Einstellungen für die folgende parlamentarische Arbeit weitgehend stabil sind. Vgl. ebenda; Helmar Schöne, a.a.O. (Fn. 15); Werner J. Patzelt, a.a.O. (Fn. 19).

42 Vgl. Renate Mayntz / Friedhelm Neidhardt, a.a.O. (Fn. 8).

43 Vgl. NRW-NI-1; NRW-NI-13; NRW-NI-38; BaWü-NI-26; BaWü-NI-43.

44 Vgl. NRW-NI-3; NRW-NI-42; NRW-NI-43; BaWü-NI-24; BaWü-NI-34.

45 Vgl. NRW-NI-17; NRW-NI-33. 
neuen Abgeordneten nicht nur als notwendige Konsequenz, sondern als erklärtes Ziel der Mandatsübernahme und der persönlichen Weiterentwicklung gesehen. ${ }^{46}$

Diesen klaren Zielen stehen jedoch nur sehr vage und zudem sehr heterogene Erwartungen zur Rolle und den Aufgaben des Abgeordneten gegenüber. Bei der Mehrheit besteht große Nervosität und Unsicherheit über ihre zukünftige Arbeit, wie das folgende Zitat eindrücklich zeigt:

„Irgendwie habe ich noch gar keine konkreten Vorstellungen davon, was mich im Landtag so erwarten wird. Seit zwei Wochen kommt aber langsam so die Nervosität und die Anspannung - man fragt sich, wie wird das so sein, wie sieht meine künftige Arbeit aus? Ich habe noch überhaupt keine Ahnung, wie das im Landtag ablaufen wird; wie ich dort Anträge stelle, wie viele Gespräche ich überhaupt haben werde. Wie viel parlamentarische Arbeit gibt es überhaupt? Komme ich noch aus dem Parlament raus? Davon habe ich noch keine Vorstellung. “47

Auffällig ist dabei, dass eine Auseinandersetzung mit der Rolle und den Aufgaben im Vorfeld kaum stattfindet, sondern dies erst nach der Wahl und somit unmittelbar vor Mandatsantritt in das Bewusstsein der neugewählten Abgeordneten tritt. Aufgrund des zumeist langjährigen kommunal- und parteipolitischen Engagements kann die große Mehrheit der neuen Abgeordneten jedoch auf vielfältige Vorerfahrungen zurückgreifen. So dienen ihnen insbesondere ihre in der Kommunalpolitik gesammelten Erfahrungen als Orientierungspunkt für die Abläufe und Aufgaben im Landesparlament und als geradezu unerlässliche Qualifikation für das Landtagsmandat.

„Vom Grundprinzip stelle ich mir die Arbeitsweise der Fraktion und des Parlaments so vor, wie es hier im Rat in (...) ist - nur, dass es um mehr Geld geht und eventuell hitziger diskutiert werden wird. “ 48

Dennoch gehen sie davon aus, dass die Strukturen, Prozesse und Mechanismen im Landesparlament eine „andere Dimension“ 49 haben als auf der kommunalen Ebene. Daher erwarten sie eine Eingewöhnungs- und Lernzeit im Landtag, über die sie jedoch im Vorfeld keine konkreten Vorstellungen und Erwartungen haben.

Einen Sonderfall nimmt hierbei jedoch circa ein Fünftel der befragten Neuparlamentarier aus Nordrhein-Westfalen und Baden-Württemberg ein. Diese waren bereits einige Jahre ,in der zweiten Reihe' des Landtags als Referenten und wissenschaftliche Mitarbeiter tätig, bevor sie selbst ein Mandat anstrebten. Durch diesen besonderen Rekrutierungspfad ${ }^{50}$ verfügen diese neuen Abgeordneten aus der Perspektive der parlamentarischen Sozialisation über spezifische Vorerfahrungen: Sie verfügen bereits über weitreichende Kenntnisse der grundsätzlichen Abläufe und Entscheidungsfindungsmechanismen im Parlament. ${ }^{51}$ Darüber hinaus haben sie bereits ein persönliches Kontaktnetzwerk zu den zentralen Akteuren des landespolitischen Betriebs etabliert. Dennoch erwartet und akzeptiert diese Gruppe

46 Vgl. NRW-NI-9; NRW-NI-16; NRW-NI-30; BaWü-NI-6; BaWü-NI-48.

47 NRW-NI-44.

48 NRW-NI-44; vgl. auch NRW-KI-8; NRW-KI-10; NRW-KI-12.

49 NRW-NI-27.

50 Vgl. Heinrich Best / Stefan Jahr / Lars Vogel, Karrieremuster und Karrierekalküle deutscher Parlamentarier, in: Michael Edinger / Werner J. Patzelt (Hrsg.), Politik als Beruf, PVS Sonderheft 44, Wiesbaden 2011; Jens Borchert / Klaus Stolz, a.a.O. (Fn. 25); Helmar Schöne, a.a.O. (Fn. 13).

51 Vgl. NRW-NI-28. 
ebenfalls eine Lernphase. Somit stellt eine vorherige Mitarbeitertätigkeit zwar einen Sozialisationsvorteil dar, aber eben keinen Sozialisationsersatz.

Hinsichtlich der Erwartungen zu ihrem eigenen Gestaltungsspielraum in der parlamentarischen Arbeit zeigt sich ein breites Spektrum, wobei sich die unterschiedlichen Erwartungshaltungen nach bisherigen Analysen überraschenderweise weder systematisch nach Art und Umfang der Vorerfahrung noch nach der Zugehörigkeit zur Regierung beziehungsweise Opposition unterscheiden ${ }^{52}$ : Eine Minderheit der neuen Abgeordneten erwartet keinen großen Gestaltungsspielraum des einzelnen Abgeordneten, insbesondere nicht in der ersten Wahlperiode. Vielmehr gehen sie davon aus, dass sie zunächst im Rahmen des Sozialisationsprozesses die Strukturen und Abläufe erlernen, Netzwerke aufbauen und sich als neue Abgeordnete beweisen müssen, wie auch die Aussage eines Abgeordneten aus Nordrhein-Westfalen verdeutlicht: „Also ich kann gut Strippen ziehen, im Job und auch in der Politik. Nur: Ich kenne hier ja noch gar keine Strippen, an denen ich ziehen kann."53

Die meisten neuen Parlamentarier gehen jedoch sehr optimistisch an die neue Aufgabe heran und erwarten, dass sie ihre inhaltlichen Ziele und Vorhaben bereits im Laufe der ersten Wahlperiode anstoßen oder realisieren können. Insofern zeigt sich hier also ein deutliches Spannungsverhältnis: Die Mehrheit der neuen Abgeordneten hat klare inhaltliche Ziele, aber nur ungenaue Vorstellungen davon, wie sie diese als Abgeordnete durchsetzen wollen beziehungsweise können. Diese Ambivalenz prägt insbesondere die erste parlamentarische Sozialisationsphase, wird als solches von den neuen Abgeordneten aber nicht wahrgenommen. Dagegen artikulieren sie jedoch eine andere Herausforderung sehr deutlich: So ist ihnen bewusst, dass mit ihrer Wahl ein Statuszuwachs verbunden ist und sie nun als Landtagsabgeordnete Teil der politischen Elite sind. ${ }^{54}$ Viele sehen es als persönliche Herausforderung, sich durch diese Position und den Sozialisationsprozess charakterlich nicht zu stark zu verändern:

„Ja, ich habe Angst davor. Also ganz ehrlich. Eine meiner Unterstützerinnen (...) hat mir geschrieben, nachdem ich gewonnen hatte: ,Bleib so wie du bist.' Das kriegen Menschen mit, wenn man sich verändert. Das ist mein Ziel, ich will so bleiben wie ich bin. Natürlich werde ich mich verändern, aber eben, dass ich meinen Charakter behalte. " 55

Dies verdeutlicht einen latenten Konflikt, in dem sich die neuen Abgeordneten sehen: Sie erwarten, dass sie sich durch die parlamentarische Sozialisation in ihren Einstellungen, Positionen und Verhaltensweisen verändern werden - gleichzeitig befürchten sie diese individuellen Veränderungsprozesse.

\section{Komponenten der Parlamentskultur: Verhaltenserwartungen und Spielregeln}

In der parlamentarischen Anfangszeit geht es - neben dem Erlernen des praktischen Knowhows - für neue Abgeordnete um die Verinnerlichung alltäglicher Routinen und informeller Spielregeln, die für das parlamentarische Handeln von zentraler Bedeutung sind. Die

53 NRW-NI-29.

54 Vgl. NRW-NI-27.

55 NRW-NI-31. 
Interviews liefern bereits deutliche Anhaltspunkte für die Existenz eines informellen Verhaltenskodex' in den untersuchten Landtagen. ${ }^{56}$ Diesbezüglich kann zwischen drei verschiedenen Handlungskontexten - der Fraktion, den interfraktionellen Beziehungen sowie dem Plenum - mit jeweils unterschiedlichen Normen differenziert werden:

Die wichtigsten Normen und informellen Spielregeln bestehen innerhalb der einzelnen Fraktionen. In den fraktionsinternen Sitzungen und Zusammenkünften werden die neuen Abgeordneten auch zum ersten Mal mit den spezifischen parlamentarischen Gepflogenheiten konfrontiert. Die erfahrenen Fraktionskollegen erwarten von den Neulingen nach wie vor zu Beginn des Mandats die Einhaltung einer Lernzeit und eine damit verbundene $\mathrm{Zu}-$ rückhaltung - zumindest so lange, bis sie ihren Platz und ihre Rolle im Parlament, in der Fraktion und den Ausschüssen gefunden haben. So haben die „alten Hasen“ selbst im Rahmen ihrer Anfangszeit diese Lernzeit eingehalten und formulieren diese Erwartung nun auch an die neuen Abgeordneten. Wie das Eingangszitat dieses Beitrags zeigt, wird das unaufgeforderte Reden ebenso wie ein zu selbstbewusstes Auftreten der Neulinge als „Sünde“ wider diese zentrale Unauffälligkeitsnorm empfunden. ${ }^{57}$

Für neue und erfahrene Abgeordnete gleichermaßen gelten grundsätzliche Verhaltensregeln, wie die Einhaltung der Fraktionsdisziplin bei Abstimmungen und der vertrauliche Umgang mit Fraktionsinterna. Für Neuparlamentarier gehört die Einschätzung, wo die Grenzen der Geheimhaltungspflicht liegen und in welchem Stadium Informationen an die Presse getragen werden dürfen, mit zum Lernprozess. Die internen Loyalitätsnormen sollen die Beziehungen zwischen den Fraktionskollegen durch Reziprozitätserwartungen stabilisieren und die Rolle des einzelnen Abgeordneten als Mannschaftsspieler definieren. Diese Anforderungen stehen dabei jedoch in einem latenten Konflikt zu anderen Erwartungen an das Verhalten der Mandatsträger: So müssen sie für ihre Wähler und ihren Wahlkreis sichtbar sein und klare Positionen vertreten, die auch im Widerspruch zur Fraktionsposition stehen können. Eine öffentliche Profilierung steht zudem der Unauffälligkeitsnorm in der Lernzeit entgegen. ${ }^{58}$

Auch für die interfraktionelle Zusammenarbeit sind Fairness-Regeln, die Verlässlichkeit von Absprachen und die damit verbundene Erwartbarkeit von Handlungen wichtige Normen. Dabei können diese in einem Spannungsverhältnis zu eher strategischen Handlungsorientierungen, wie der Logik der Mehrheitsbeschaffung für eigene Gesetzesvorhaben, stehen. Der parteiübergreifende Konsens über Fairness-Normen manifestiert sich immer dann besonders klar, wenn er durch das Verhalten einzelner Abgeordneter oder ganzer Fraktionen in Frage gestellt wird. Dies wird auch an dem eingangs aufgegriffenen Beispiel der Aufkündigung des Pairing-Abkommens im Nordrhein-Westfälischen Landtag im Rahmen der Abstimmung zum WestLB-Paket deutlich. In den an diesem und in den Folgetagen geführten Interviews wurde dies fraktionsübergreifend als Verstoß gegen die „parlamentarische Fairness "59 und als Verletzung einer elementaren Handlungsnorm des kollegialen Miteinanders bewertet. Auch die neuen Abgeordneten, denen diese interfraktionelle Spielregel häufig bis zum Zeitpunkt ihrer Verletzung unbekannt war, sahen dadurch die interfraktionelle Vertrauensbasis nachhaltig beschädigt. ${ }^{60}$

56 Siehe grundlegend Ulrich Sarcinelli, a.a.O. (Fn. 11), S. 402 f.

57 Vgl. NRW-KI-10; NRW-KI-12; BaWü-KI-8.

58 Vgl. NRW-NII-42; BaWü-NI-27.

59 Bernd Dörries, a.a.O. (Fn. 4).

60 Vgl. NRW-NII-44; NRW-NII-4; NRW-NII-10; NRW-NII-20; NRW-NII-25; NRW-NII-39. 
Für das Verhalten im Plenarsaal als öffentlicher Komponente parlamentarischen Handelns sind wiederum andere Orientierungen und Spielregeln von besonderer Bedeutung. Neben den formalen, in der Geschäftsordnung festgehaltenen Verhaltensregeln gehören die Vermeidung demonstrativer Unaufmerksamkeit und persönlicher Angriffe sowie eine angemessene Ausdrucksweise zum informellen Verhaltenskodex und zum gemeinsamen Verständnis von der „Würde des Hohen Hauses“. Auch zeigt sich in beiden untersuchten Landtagen der fast einhellige Konsens, dass das Parlament ein „Ort der Debatte, nicht der Demonstration“ sei. Des Weiteren existieren kollektive Vorstellungen darüber, wie sich ein Abgeordneter zu kleiden hat, obwohl darin kein großes Konfliktpotenzial (mehr) gesehen wird. Dennoch scheint, dass zu den verhaltensbezogenen Selbstverständlichkeiten gerade auch die Wahl „angemessener“ Kleidung und die Reflektion der repräsentativen Rolle im äußeren Erscheinungsbild gehören.

Aktueller als die (klassischen) Kleiderfragen sind heutzutage Diskussionen über die Veränderung der parlamentarischen Kultur durch die Nutzung neuer Medien im Plenarsaal. War im Düsseldorfer Landtag der Einsatz von Informationstechnologien bisher grundsätzlich untersagt, konnten vor allem jüngere Abgeordnete im Mai 2011 eine Lockerung des Technikverbots durchsetzen, die sich an der im Bundestag getroffenen Regelung orientiert. Seither ist die Nutzung von Computern erlaubt, die keinen aufklappbaren Bildschirm haben (TabletPCs) und geräuschlos bedient werden können. Im Baden-Württembergischen Landtag zeichnet sich derzeit eine ähnliche Regelung ab. ${ }^{61}$ Dies verweist auf das interdependente Verhältnis von Parlamentsnormen und Abgeordneten. Nicht nur die Neulinge passen sich an die informellen Spielregeln an, sondern auch die Normen und Spielregeln unterliegen einem Wandel.

Neben diesen Sozialisationsprozessen auf der individuellen Ebene konnte anhand der 2010 erstmals in den Nordrhein-Westfälischen Landtag eingezogenen Fraktion der Linken untersucht werden, wie die parlamentarische Sozialisation auf kollektiver Ebene verläuft. Ähnlich wie die Grünen in den 1980er Jahren verstieß auch die Linksfraktion gezielt mit einigen Aktionen gegen parlamentsinterne Normen. ${ }^{62}$ Gleichzeitig ist den Abgeordneten jedoch bewusst, dass ein allzu häufiger Regelbruch und die Nutzung des Plenarsaals als „Ort der Demonstration“ die Durchsetzung ihrer politischen Ziele langfristig erschwert. ${ }^{63}$ Daher deutet sich hier an, dass es im Spannungsfeld zwischen politischen Differenzen und institutionellen Normen auch auf kollektiver Ebene zu einer Vermittlung durch die Prozesse parlamentarischer Sozialisation kommt.

\section{Vermittler der Parlamentskultur: Sozialisationsagenten}

Sucht man nach den Vermittlern der zentralen Normen und informellen Spielregeln, so zeigt sich, dass insbesondere die erfahrenen Fraktionskollegen eine entscheidende Rolle bei

61 Vgl. NRW-NII-9; BaWü KI-13. Siehe auch Tobias Blasius, Landtag streitet um Laptop und iPad, 31. Januar 2011, http://www.derwesten.de/nachrichten/Landtag-streitet-um-Laptop-und-iPadid4228820.html (Abruf am 22. August 2011).

62 Dies zeigte sich beispielsweise auch in der konstituierenden Sitzung des Nordrhein-Westfälischen Landtags als die Abgeordneten der Linksfraktion mit gelben Bildungsstreikshirts den Plenarsaal betraten. Siehe auch „NRW hat einen neuen Landtag“, 9. Juni 2010, http://www.derwesten.de/ nachrichten/NRW-hat-einen-neuen-Landtag-id3320041.html (Abruf am 23. August 2011).

63 Vgl. NRW-NI-32; NRW-NI-47. 
der Vermittlung der zentralen Normen und informellen Spielregeln einnehmen. Dabei verfügt ein Teil der neu eingezogenen Abgeordneten bereits vor Mandatsantritt über engere Kontakte zur Fraktion. Als wichtige Ansprechpartner fungieren meistens die Parlamentarier aus der gleichen Region sowie persönliche Bekannte aus Parteigremien oder Vorfeldorganisationen (wie die Jugend- und Frauenorganisationen). Die Vermittlung der zentralen Spielregeln erfolgt dabei häufig in Form von kollegialen Ratschlägen und Hinweisen, insbesondere zur öffentlichen Rolle und medialen Beobachtung. Hierbei stehen einzelne erfahrene Fraktionskollegen den Neuen als selbsternannte „Experten“ unterstützend zur Seite. Eine SPD-Abgeordnete aus Baden-Württemberg hat sich innerhalb ihrer Fraktion als Ansprechpartnerin für die bereits angeführten Kleidungsfragen etabliert:

„(...) Man hat mich so scherzeshalber zur kleidungspolitischen Beauftragten der Fraktion gemacht. Ich gucke dann auch, dass gerade unsere Jungen, die da gekommen sind, dass die sich ordentlich anziehen bei bestimmten Anlässen. So Kleinigkeiten. Die würden sich nämlich hier unwohl fühlen, wenn sie plötzlich merken würden: Ey, ich bin ja der Einzige hier, der in Jeans und Hemd 'rumrennt." "64

Als weitere relevante Sozialisationsfelder wurden zudem die sowohl im Düsseldorfer als auch im Stuttgarter Landtag existierenden regionalen Untergruppierungen innerhalb der Fraktionen ausgemacht. Diese dienen der gemeinsamen regionenspezifischen Interessenvertretung in der Fraktion und im Parlament und nehmen dabei gleichzeitig eine wichtige Vermittlungsrolle für die neuen Abgeordneten ein. Ein Beispiel ist das „Teutonenfrühstück“, bei dem sich die sozialdemokratischen Abgeordneten der Region Ostwestfalen-Lippe regelmäßig während der Plenartage zum Arbeitsfrühstück mit eigener Tagesordnung treffen. ${ }^{65}$

Wenn die traditionellen Sozialisationsagenten nicht (ausreichend) vorhanden sind, werden zur Kompensation der fehlenden - meist fraktionellen - Organisations- und Wissensstrukturen parlamentsexterne Sozialisationsagenten beauftragt. Diese Vermittler der parlamentarischen Spielregeln haben keine Kenntnisse der organisationsspezifischen Parlamentskultur, verfügen jedoch über allgemeine Expertise zu den Strukturen und Spielregeln parlamentarischer Arbeit. So erhielt etwa die Linke bei ihrem erstmaligen Einzug in den Düsseldorfer Landtag beim Fraktionsaufbau „Entwicklungshilfe aus dem Osten“66. Diese leistete ein parteiinterner Berliner Politikberater, der auch in anderen westlichen Landesverbänden den Aufbau neuer linker Fraktionen beratend und unterstützend begleitete, wie ein Abgeordneter erläutert:

„Wir haben auch Unterstützung aus Berlin; es gibt da jemanden, der uns begleitet - es gibt ja bisher noch keine Erfahrungen der Linken beim Fraktionsaufbau im Westen. Allerdings sind wir mittlerweile in jedem Kreistag und jedem Stadtrat in NRW vertreten. Wir machen also bereits parlamentarische Arbeit. Aber diese Person berät uns bei allem, was nun wichtig ist

64 BaWü-KI-9.

65 Vgl. NRW-NII-39.

66 Hier zeigt sich insofern also ein Ost-West-Transfer zur Unterstützung des Aufbaus der westdeutschen Linken. Dies ist insbesondere vor dem Hintergrund des West-Ost-Transfers politischer Institutionen zur Unterstützung des ostdeutschen Transformationsprozess nach der Wiedervereinigung bemerkenswert; vgl. Christian Welzel, Rekrutierung und Sozialisation der ostdeutschen Elite, in: Wilhelm Bürklin / Hilke Rebenstorf (Hrsg.), Eliten in Deutschland. Rekrutierung und Integration, Opladen 1997, S. $201-237$. 
und entschieden werden muss - ich sage bewusst Beratung; wir entscheiden, aber er sagt uns, worüber wir sprechen sollten. "67

Auch die Fraktion der Grünen in Baden-Württemberg beauftragte parlamentsexterne Sozialisationsagenten, um ein asymmetrisches Verhältnis zwischen erfahrenen Abgeordneten als Repräsentanten der sozialisierenden Institution und den Neuen innerhalb der Fraktion auszugleichen. Nach dem Wahlsieg im Mai 2011 sind zwei Drittel der Fraktionsmitglieder parlamentarische Neulinge, die nicht allein durch die eigenen fraktionsinternen Sozialisationsagenten integriert werden können. Zur Unterstützung der parlamentarischen Sozialisation und zur Definition der neuen Rolle als Regierungsfraktion beauftragte die Fraktion daher die Weiterbildungsagentur GreenCampus der Heinrich-Böll-Stiftung ${ }^{68}$ :

„Es gibt so informelle Patenschaften, die wir Alten für die Neuen übernommen haben (...), und wir planen jetzt einen ganztägigen Workshop. Die Alten und die Neuen zusammen, um gemeinsam zu diskutieren: Was ist die Rolle eines Abgeordneten von der Regierungsfraktion? Wir haben eine externe Fraktionsentwicklungsagentur, also GreenCampus (...). Also wir haben ja alle auf einmal Führungsaufgaben, und für viele ist das neu. Und jetzt ist natürlich auch zu klären, welchen Stellenwert, welche Aufgaben haben parlamentarische Berater, was macht ein Abgeordneter, wie funktioniert die Vernetzung, wie sind die Hierarchien. Also dieses ganze Soziogramm zu besprechen. “69

\section{Neue Abgeordnete treffen aufstarke Normen der Parlamentskultur}

Parlamente sind besondere Organisationen: So wechselt periodisch ein Teil der Angehörigen, wobei die Organisation selbst keinen Einfluss auf die Auswahl der Neuen hat. Trotz oder gerade wegen dieser regelmäßigen Fluktuation führen Parlamente ein starkes organisatorisches Eigenleben. Sie haben eine jeweils eigene Kultur, die nicht mehr hinterfragte Routinen alltäglichen Handelns begründet.

Auf diese Parlamentskultur treffen die neuen Abgeordneten je nach Vorerfahrungen mehr oder weniger unvorbereitet. Innerhalb kurzer Zeit müssen sie sich auf die herrschenden Spielregeln einstellen. Anders als etwa die Geschäftsordnung des Landtages, die man nachlesen kann, sind die meisten dieser Spielregeln zum einen informell und ungeschrieben, zum anderen oft so tief verwurzelt, dass sie den erfahrenen Abgeordneten zum Teil selbst gar nicht mehr ständig bewusst sind. Häufig ist es erst die Verletzung einer Spielregel, durch die deren Gültigkeit und Bedeutung klar wird.

Während die Funktion einer fraktionsübergreifenden Parlamentskultur relativ klar ist - sie soll eine verlässliche Kooperation von Abgeordneten ermöglichen, die als Angehörige konkurrierender Parteien immer auch im Wettbewerb miteinander stehen -, sind die Vermittlungsmechanismen, durch die neue Abgeordnete die herrschenden Regeln erlernen und sich (überwiegend) an sie anpassen, weitaus weniger deutlich. Für die beiden untersuchten Landtage in Nordrhein-Westfalen und Baden-Württemberg lassen sich insbesondere für die frühe Phase der parlamentarischen Sozialisation neuer Abgeordneter drei Feststellungen treffen:

67 NRW-NI-47; vgl. auch NRW-NI-40; NRW-NI-46.

68 Vgl. BaWü-KI-8; BaWü-KI-14.

69 BaWü-KI-8. 
(1) Die neuen Abgeordneten ziehen einerseits mit klaren und häufig konkreten inhaltlichen Zielen erstmals in das Parlament ein. Diesen klaren Zielen stehen andererseits jedoch interessanterweise unklare und heterogene Erwartungen zur Rolle und den Aufgaben des Abgeordneten gegenüber. Entsprechend haben die meisten Neuen nur eine sehr vage Vorstellung davon, wie sie ihre inhaltlichen Ziele durchsetzen wollen beziehungsweise können. Dieses Spannungsverhältnis prägt die erste parlamentarische Sozialisationsphase.

(2) Die bisherigen Ergebnisse der Studie weisen auf wenige, jedoch starke und sanktionsbewehrte Normen und informelle Spielregeln hin, die den Rahmen für das Verhalten und Handeln der Abgeordneten bilden. Zu diesen Normen gehören die fraktionsinterne Loyalität und die fraktionsübergreifende Fairness. Speziell von den neuen Abgeordneten wird zudem in der Anfangszeit eine gewisse Zurückhaltung in der Fraktion und im Parlament erwartet. Aus Sicht der Neuen ist häufig jedoch unklar, welches Verhalten die einzelnen Normen von ihnen erfordern, die zudem allesamt in einem latenten Spannungsverhältnis zu den bereits im Vorfeld selbstgesteckten Zielen und Motiven stehen, da sie die Handlungsfreiheit der nunmehr gewählten Abgeordneten beschränken.

(3) Es zeigt sich eine Dominanz der traditionellen fraktions- und parteiinternen Sozialisationsagenten und somit wenig Modernisierung. Insbesondere die erfahrenen Fraktionskollegen spielen nach wie vor eine sehr wichtige Rolle als Vermittler der Normen und informellen Spielregeln. Im Kontext gegenwärtiger gesellschaftlicher Entwicklungen erscheint es zunächst überraschend, dass kaum professionelle externe Berater herangezogen werden und es auch keine Ansätze einer institutionalisierten Einführung in das Parlamentarierdasein gibt.

Diese ersten Ergebnisse aus der frühen parlamentarischen Sozialisationsphase der neuen Landtagsabgeordneten werden im Rahmen des Gesamtprojekts zu erweitern und systematisch zu analysieren sein. Wie gezeigt, verfügen die Neuen über sehr unterschiedliche Erfahrungen und ziehen mit sehr unterschiedlichen Vorstellungen und Einstellungen erstmals in den Landtag ein. Insofern bleibt die Frage im Zentrum, wie sich die Abgeordneten und ihre parlamentsbezogenen Einstellungen und Verhaltensweisen durch die Sozialisationsprozesse im Spannungsfeld zwischen den Anforderungen innerhalb und außerhalb des Parlaments verändern und ob Prozesse der Konvergenz und Introversion zu beobachten sind.

Die hier vorgestellten Analysen zur Sozialisation in die Parlamentskultur stellen die Gemeinsamkeiten der Prozesse in den beiden Landtagen in den Vordergrund. Offen ist noch, ob und inwiefern es Unterschiede zwischen den beiden gibt. Diese sollen systematisch daraufhin untersucht werden, welchen Einfluss die institutionellen Rahmenbedingungen wie Wahlsystem, Parteiensystem, Mehrheitsverhältnisse und vorherrschende Karrierestrukturen auf die Sozialisationsprozesse haben.

Ein besonderer Schwerpunkt soll schließlich auf der Frage liegen, ob sich die zentralen Normen und Spielregeln als Ausdruck der Parlamentskultur verändern. Einerseits könnte dies eine Folge der veränderten Konstellationen in den beiden untersuchten Landtagen nach den Landtagswahlen sein - die grün-rote Mehrheit und der erste grüne Ministerpräsidenten in Baden-Württemberg sowie die unklaren Mehrheitsverhältnisse und die rot-grüne Minderheitsregierung in Nordrhein-Westfalen. Anderseits könnte es eine Reaktion auf allgemeinere Veränderungstendenzen im politischen Wettbewerb sein. Hierfür spräche, dass die Pairing-Regel jüngst auch in anderen Landtagen in Frage gestellt wurde. 